\title{
Sacral Insufficiency Fractures are Common After High-dose Radiation for Sacral Chordomas Treated With or Without Surgery
}

\author{
Polina Osler MS, Miriam A. Bredella MD, Kathryn A. Hess BS, \\ Stein J. Janssen MD, Christine J. Park BS, Yen Lin Chen MD, \\ Thomas F. DeLaney MD, Francis J. Hornicek MD, PhD, Joseph H. Schwab MD, MS
}

Received: 7 April 2015/Accepted: 16 September 2015/Published online: 23 September 2015

(C) The Association of Bone and Joint Surgeons (B) 2015

\begin{abstract}
Background Surgery with high-dose radiation and highdose radiation alone for sacral chordomas have shown promising local control rates. However, we have noted frequent sacral insufficiency fractures and perceived this rate to be higher than previously reported.

Questions/purposes We wished (1) to characterize the incidence of sacral insufficiency fractures in patients with
\end{abstract}

One author (SJJ) certifies that he has received, an amount less than USD 10,000 from the Anna Foundation (Oegstgeest, The Netherlands), an amount less than USD 10,000 from the De Drie Lichten Foundation (Hilversum, The Netherlands), an amount less than USD 10,000 from the KWF Kankerbestrijding (Amsterdam, The Netherlands), and an amount less than USD10,000 from the Michael van Vloten Foundation (Rotterdam, The Netherlands). One author (JHS) certifies that he or a member of his family has or may receive payments or benefits, an amount less than USD 10,000 from Stryker (Kalamazoo, MI, USA), and an amount less than USD 10,000 from Biom'up (Saint-Priest, Lyon, France).

All ICMJE Conflict of Interest Forms for authors and Clinical Orthopaedics and Related Research ${ }^{\circledR}$ editors and board members are on file with the publication and can be viewed on request.

Each author certifies that his or her institution approved or waived approval for the reporting of this investigation and that all investigations were conducted in conformity with ethical principles of research.

This work was performed at Massachusetts General Hospital, Boston, MA, USA.

P. Osler

Harvard Medical School, Boston, MA, USA

M. A. Bredella

Musculoskeletal Imaging and Interventions, Department of Radiology, Massachusetts General Hospital, Boston, MA, USA

K. A. Hess

Department of Orthopaedic Surgery, Tufts Medical Center, Boston, MA, USA chordomas of the sacrum who received high-dose radiation, and (2) to determine whether patients treated with surgery plus high-dose radiation or high-dose radiation alone are more likely to experience a sacral fracture, and to compare time to fracture in these groups.

Methods Sixty-two patients who received high-dose radiation for sacral chordomas with $(n=44)$ or without surgical resection $(n=18)$ between 1992 and 2013 were included in this retrospective study. At our institution, sacral chordomas generally are treated by preoperative radiotherapy, followed by en bloc resection, and postoperative radiotherapy. Radiation alone, with an intent to cure, is offered to patients who otherwise are not good surgical candidates or patients who elect radiotherapy based on tumor location and the anticipated morbidity after surgery (such as sexual, bowel, or bladder dysfunction). MRI and CT scans were evaluated for evidence of sacral insufficiency fractures. Complete followup was available at a minimum of 2 years (or until fracture or death) for all 18 patients who underwent radiation alone, whereas $14 \%$ (six of 44 patients) in the surgery plus radiation group (9\% [three of 33] after high sacrectomy and 27\% [three of 11] after low sacrectomy) were lost to followup before 2 years. Results Sacral insufficiency fractures occurred in 29 of the 62 patients (47\%). A total of 25 of 33 patients (76\%)

S. J. Janssen ( $₫)$, C. J. Park, F. J. Hornicek, J. H. Schwab Department of Orthopaedic Surgery, Orthopaedic Oncology Service, Massachusetts General Hospital, 55 Fruit Street, Boston, MA 02114, USA

e-mail: steinjanssen@gmail.com

Y. L. Chen, Thomas F. DeLaney

Department of Radiation Oncology, Massachusetts General Hospital, Boston, MA, USA 
with high sacrectomy had fractures develop compared with zero of $11(0 \%)$ after low sacrectomy, and four of the 18 patients $(22 \%)$ who had high-dose radiation alone $(\mathrm{p}<$ $0.001)$. The fracture rate was greater in the high sacrectomy group than in the low sacrectomy group $(\mathrm{p}<0.001)$ and the radiation only group $(\mathrm{p}<0.001)$. There was no difference with the numbers evaluated in fracture probability between patients in the low-sacrectomy group and those treated with radiation alone $(\mathrm{p}=0.112)$. The fracture-free survival probability was 0.99 for the low sacrectomy group at all times as there were no insufficiency fractures in this group; the 1-year fracture-free survival probability was $0.53(95 \%$ CI, 0.35-0.69) after high sacrectomy, 0.83 (95\% CI, 0.570.94 ) after radiation alone; the 2-year fracture-free survival probability was 0.36 (95\% CI, 0.19-0.52) after high sacrectomy and 0.77 (95\% CI, 0.50-0.91) after radiation alone; and the 5-year fracture-free survival probability was 0.14 (95\% CI, 0.04-0.30) after high sacrectomy and 0.77 (95\% CI, 0.50-0.91) after radiation alone.

Conclusions Acknowledging the limitations of potential differences in baseline and followup among treatment groups in our study, we found that almost $1 / 2$ of our patients experienced an insufficiency fracture. We found that the fracture rate was greater in the surgery group compared with the radiation alone group and that high sacrectomy accounted for all fractures in the surgery group. These findings can be used to inform patients and also support the need for further research to elucidate the influence of highdose radiation on bone quality.

Level of Evidence Level III, therapeutic study.

\section{Introduction}

At our institution, the most common primary malignant bone tumor of the sacrum is a chordoma. The standard of care for treatment of a sacral chordoma is en bloc resection. In the spine, external beam radiation therapy combined with surgical resection has been shown to be an effective option for treatment of historically radiationresistant tumors [2, 13]. We have combined surgery with high-dose proton-based radiation with good results, with 94\% local tumor control at 5 years for chordomas and other malignant bone tumors in the sacrum [4]. We also have had good results after high-dose radiation alone: $80 \%$ local tumor control at 5 years in patients who elected nonsurgical treatment or patients who otherwise were not good surgical candidates [2].

Surgery with high-dose radiation and radiation alone have shown promising local control rates. However, we have observed frequent sacral insufficiency fractures associated with this treatment. Previous studies reported a $6 \%$ sacral insufficiency rate after surgical treatment of sacral chordomas [6, 14], however we believe our rate is higher than those reported.

We aimed (1) to characterize the incidence of sacral insufficiency fractures in patients with chordomas of the sacrum who received high-dose radiation, and (2) to determine whether patients treated with surgery plus highdose radiation or high-dose radiation alone are more likely to experience a sacral fracture, and to compare time to fracture in these groups.

\section{Patients and Methods}

\section{Study Design, Setting, and Participants}

Our institutional review board approved this retrospective study and waived informed consent. We reviewed medical records of all patients with a sacral chordoma who presented to the Orthopaedic Oncology Service of Massachusetts General Hospital between 1992 and 2013. Sixty-two patients who were 18 years or older who received high-dose radiation (more than $50 \mathrm{~Gy}$, combination of protons and photons) to the sacrum with curative intent were included in the study. Patients with sacral insufficiency fractures before treatment, patients who underwent a hemisacrectomy or sacroiliac joint resection, and those who did not receive the planned highdose radiation (eg, owing to wound complications or patient preference) were excluded. Clinic notes were reviewed to collect demographic information including sex, age at treatment, and tumor diagnosis.

\section{Treatment}

At our institution, sacral chordomas generally are treated by preoperative radiotherapy, followed by en bloc resection, and postoperative boost radiotherapy. Radiation alone (with an intent to cure) is offered to patients who otherwise are not good surgical candidates (eg, patients with severe cardiopulmonary comorbidities) or patients who elect nonsurgical management based on tumor location and the anticipated morbidity after surgery (eg, sexual, bowel, and voiding dysfunction). High-dose radiation is the standard radiotherapy regimen for the surgery and the radiation only group. The number of patients who underwent surgical resection without high-dose radiation is low, and this is discussed only with patients with caudally located tumors without soft tissue invasion. However, even in this situation our recommended protocol is radiation followed by surgery and then additional radiation. Whenever possible, wide margin resection of the tumor was attempted in patients undergoing surgery. For this study, we divided surgical patients into high sacrectomy (at S3 level or above) and low 
sacrectomy (below S3 level) groups, depending on the type of osteotomy that was performed. The operative notes were reviewed by the senior orthopaedic surgeon JHS to record the level of the sacral osteotomy. Information regarding the radiation regimen and doses was reviewed by the senior radiation oncologist (TFD). In our cohort, 15 of the 18 patients who received radiation alone declined surgical resection based on the anticipated morbidity, one patient declined transfusions and therefore was offered radiation. The reason for radiation alone was unclear in two patients.

\section{Outcome Assessment: Sacral Insufficiency Fracture}

All patients had at least one pretreatment MRI, and were evaluated routinely with followup imaging at least

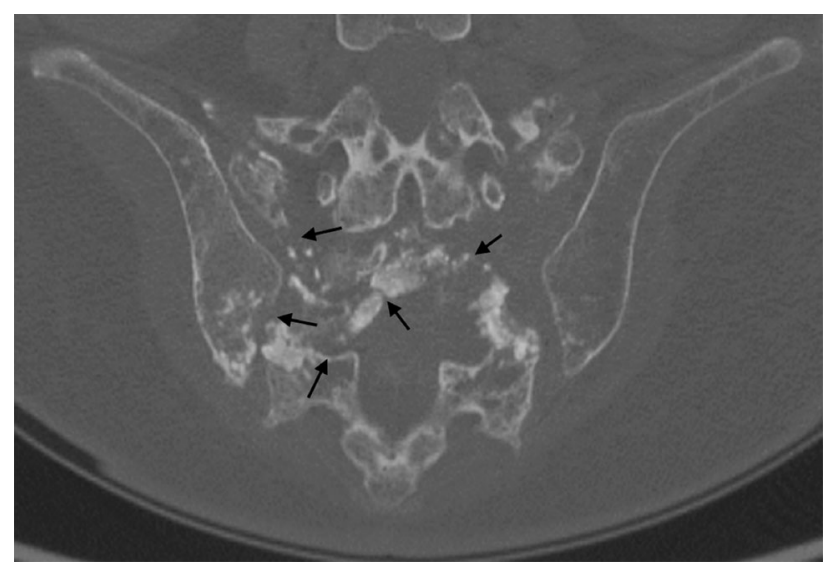

Fig. 1 A 64-year old woman with a history of chordoma experienced radiation necrosis with sacral insufficiency fractures as seen on this CT scan. The coronal image shows postsurgical changes from a partial sacrectomy and extensive destruction, sclerosis, and fragmentation of the sacrum, consistent with radiation necrosis. Multiple sacral insufficiency fractures are present (black arrows). annually. In general, a CT scan of the pelvis was ordered only if there was clinical concern for fracture (Fig. 1). All imaging studies were reviewed by the senior radiologist (MAB) for presence of sacral insufficiency fractures. The definition of an insufficiency fracture is a fracture attributable to normal stress on abnormal bone and the imaging criteria are the same as for any other fracture; areas with linear decreased T1- and increased T2-weighted signals in the sacrum observed on MRI were considered to be indicative of insufficiency fractures (Fig. 2) [20]. CT scans can show fractures, but also can appear normal; therefore we used MR images, if they were available.

\section{Statistical Analysis}

Variables were presented with frequencies and percentages for categorical variables and as median with range for continuous variables, as visual inspection of histograms suggested nonnormality. Fracture-free survival was calculated using Kaplan-Meier analysis.

In bivariate analysis, the differences between baseline treatment groups were compared using a Kruskal-Wallis test (ANOVA-on-ranks) for continuous variables, and Fisher's exact test for categorical variables.

Fracture-free survival times between treatment groups were compared using the log-rank test. We used KaplanMeier plots to show the fracture-free survival per treatment group. Visual inspection of log-log plots suggest no serious violation of the proportional hazards assumption [1]. A two-sided $\mathrm{p}$ value less than 0.05 was considered statistically significant. Statistical analysis was performed using Stata ${ }^{\circledR} 14.0$ (StataCorp LP, College Station, TX, USA). The exact radiation dose was unavailable for one patient who underwent surgery, and for four patients who received
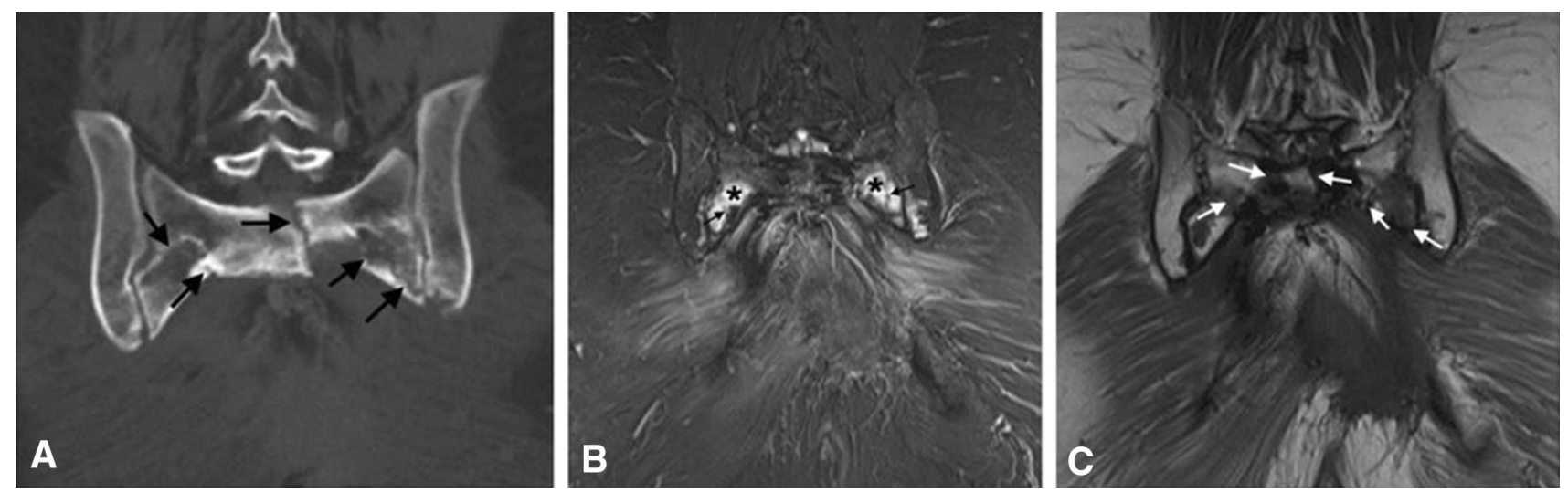

Fig. 2A-C A 47-year-old woman with a history of chordoma resection and radiation therapy had sacral insufficiency fractures after surgery. (A) The coronal CT image shows multiple sacral insufficiency fractures with adjacent sclerosis (black arrows). (B) The coronal fat-suppressed
T2-weighted image shows bone marrow edema (asterisks) adjacent to hypointense insufficiency fractures (black arrows), and (C) the coronal T1-weighted image shows hypointense linear signal abnormalities (white arrows), consistent with insufficiency fractures. 
high-dose radiation alone. There were no missing values for any of the other variables.

\section{Patient Demographics}

We included 62 patients with a median age of 61 years (range, 22-84 years). There were 37 (60\%) males and 25 $(40 \%)$ females. Preoperative diagnoses included 55 (89\%) primary sacrococcygeal chordomas, six $(9.7 \%)$ recurrent sacral chordomas after prior surgery, and one (1.6\%) chondroid chordoma. Forty-four $(71 \%)$ patients were treated with surgery and high-dose radiation. Eighteen (29\%) patients were treated with high-dose radiation alone. Of the 44 patients who underwent surgery, $33(75 \%)$ had a high sacrectomy, and $11(25 \%)$ had a low sacrectomy. Lumbopelvic reconstruction with rods and/or allograft struts was done in seven (21\%) of the 33 patients who had high sacrectomy. All patients received high doses of radiation according to the previously described protocol [5]. The median radiation dose received by all patients was 70.2 Gy (range, 50.4-79.2 Gy) and was a combination of photons and protons [2, 4]. In the surgical group, all patients received preoperative radiation (median dose, 50.4 Gy), and 40 of 44 patients (91\%) received additional postoperative boost radiation per established protocol (median dose, $19.8 \mathrm{~Gy}$ ). Three of four patients did not receive postoperative radiation owing to wound complications, and one patient rejected postoperative radiation. In the patient who had gross residual disease remaining after surgery, the tumor bed margins were given approximately 70.2 Gy and the area of gross residual disease was given approximately 77.4 Gy.

There were no differences in the age $(\mathrm{p}=0.221)$ or sex $(\mathrm{p}=0.884)$ among the treatment groups. Total radiation dose differed between groups $(\mathrm{p}<0.001)$ and was greatest in the radiation only group (median, $77.4 \mathrm{~Gy}$ ), compared with the high and low sacrectomy groups (median, 70.2 Gy for both groups) (Table 1). There was no difference in the amount of radiation received by the high sacrectomy group versus the low sacrectomy group ( $\mathrm{p}=0.368$, by MannWhitney $\mathrm{U}$ test), but both groups received less radiation than the nonsurgical group ( $\mathrm{p} \leq 0.001$, by Mann-Whitney U test).

\section{Followup}

The median followup was 22 months, with an interquartile range from 9 to 54 months.

Of the 44 patients who underwent surgery with radiation, $20(45 \%)$ had an insufficiency fracture, one (2\%) was deceased, and six (14\%) were lost to followup within 2 years. The remaining 17 (39\%) patients had more than 2 years of followup. Of the 18 patients who underwent radiation only, four $(22 \%)$ had an insufficiency fracture, one $(6 \%)$ was deceased, and none were lost to followup within 2 years. The remaining $13(72 \%)$ patients had more than 2 years of followup.

In the high sacrectomy group ( $\mathrm{n}=33), 20(61 \%)$ patients had insufficiency fractures and three (9\%) were lost to followup within 2 years. The remaining $10(30 \%)$ had more than 2 years of followup. In the low sacrectomy group $(\mathrm{n}=$ $11)$, one patient was deceased $(9 \%)$ and three $(27 \%)$ were lost to followup within 2 years. The remaining seven (64\%) had more than 2 years of followup.

\section{Results}

Almost $1 / 2$ of the patients in our cohort had a sacral insufficiency fracture (47\%; 29 of 62 patients). The overall fracture-free survival probability is 0.71 (95\% CI, 0.570.80 ) after 1 year, 0.60 (95\% CI, 0.46-0.71) after 2 years, and 0.48 (95\% CI, 0.34-0.61) after 5 years (Fig. 3).

We found a higher fracture rate after surgery with highdose radiation $(57 \% ; 25$ of 44) compared with high-dose radiation alone (22\%; four of 18$)(\mathrm{p}=0.009)$ (Fig. 4). The 1 -year fracture-free survival probability is 0.65 (95\% CI, $0.49-0.77)$ after surgery and 0.83 (95\% CI, 0.57-0.94) after radiation alone; the 2-year fracture-free survival probability is $0.52(95 \% \mathrm{CI}, 0.36-0.66)$ after surgery and 0.77 (95\% CI, 0.50-0.91) after radiation alone; and the 5-year fracture-free survival probability is 0.33 (95\% CI, 0.17-0.49)

Table 1. Patient population and demographics

\begin{tabular}{lccc}
\hline Demographics & Radiotherapy $(\mathrm{n}=18)$ & High sacrectomy $(\mathrm{n}=33)$ & Low sacrectomy $(\mathrm{n}=11)$ \\
\hline Age (years) (median/range) & $62(42-79)$ & $54(22-81)$ & $64(34-84)$ \\
Sex (number/\%) & & & \\
$\quad$ Men & $10(56)$ & $20(61)$ & $7(64)$ \\
Women & $8(44)$ & $13(39)$ & $4(36)$ \\
Radiation dose (Gy) & $77.4(75-79.2)$ & $70.2(50.4-77.6)$ & $70.2(50.4-73.8)$ \\
(median/range) & & & 0.884 \\
\hline
\end{tabular}


after surgery and 0.77 (95\% CI, 0.50-0.91) after radiation alone (Fig. 4). We also found a difference in fracture rate when comparing high-sacrectomy $(76 \% ; 25$ of 33$)$, lowsacrectomy ( $0 \%$; 0 of 11$)$, and radiation alone (22\%; four of 18) $(\mathrm{p}<0.001)$ (Fig. 5). The fracture rate was greater in the high-sacrectomy group than in the low-sacrectomy group $(\mathrm{p}<0.001)$ and the radiation only group $(\mathrm{p}<0.001)$. There was no difference with the numbers evaluated in fracture probability between patients in the low-sacrectomy group and those treated with radiation alone $(p=0.112)$. The fracture-free survival probability is 0.99 for the low sacrectomy group at all times, as there were no insufficiency fractures in this group; the 1-year fracture-free survival probability is 0.53 (95\% CI, 0.35-0.69) after high sacrectomy, and 0.83 (95\% CI, 0.57-0.94) after radiation alone. The 2-year fracture-free survival probability is 0.36 (95\% CI, 0.19-0.52) after high sacrectomy and 0.77 (95\%

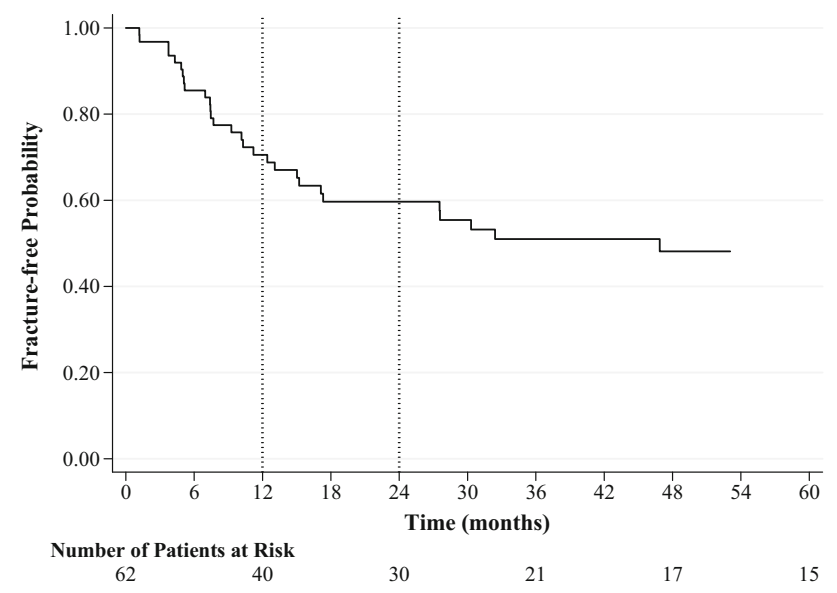

Fig. 3 Fracture-free survival probability among all patients is shown.

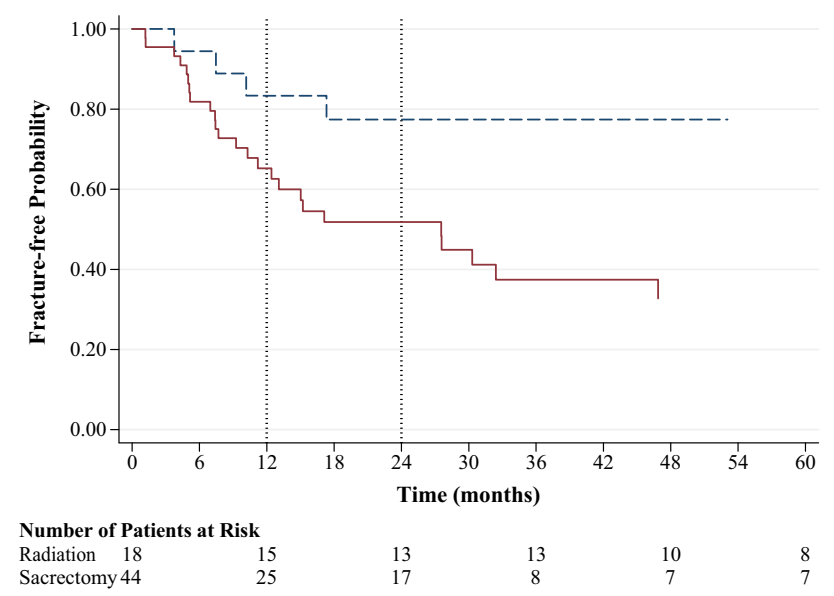

Fig. 4 Fracture-free survival probabilities in the surgical group (red solid line) compared with the nonsurgical group (blue dashed line) are shown.
CI, 0.50-0.91) after radiation alone. The 5-year fracturefree survival probability is 0.14 (95\% CI, 0.04-0.30) after high sacrectomy and 0.77 (95\% CI, 0.50-0.91) after radiation alone (Fig. 5).

We found no difference in fracture probability between males and females with the numbers evaluated $(\mathrm{p}=0.218)$ (Fig. 6).

\section{Discussion}

Surgery with high-dose radiation and radiation alone for sacral chordomas have shown promising local control rates. However, we have seen frequent sacral insufficiency fractures and perceived this rate to be higher than previously reported $[6,14]$. We therefore studied the incidence of insufficiency fractures after high-dose radiation for sacral chordomas and assessed if this rate differed between surgery plus high-dose radiation and high-dose radiation alone. We found that nearly $1 / 2$ of our patients experienced a fracture and that the fracture rate was higher in the surgery group compared with the radiation alone group.

Our study has several limitations. First, although age and sex did not differ among treatment groups, other factors, such as location and size of the tumor, might have. This selection bias, inherent to the retrospective study design, could not have been eliminated from our study, and we see this as an important limitation as these confounding factors might have influenced the sacral insufficiency rate overall and in treatment groups. Second, our outcome measure was sacral insufficiency fracture detected on MRI or CT and we did not address associated symptoms, hampering assessment of its clinical relevance. Sacral insufficiency fractures can cause debilitating low back pain

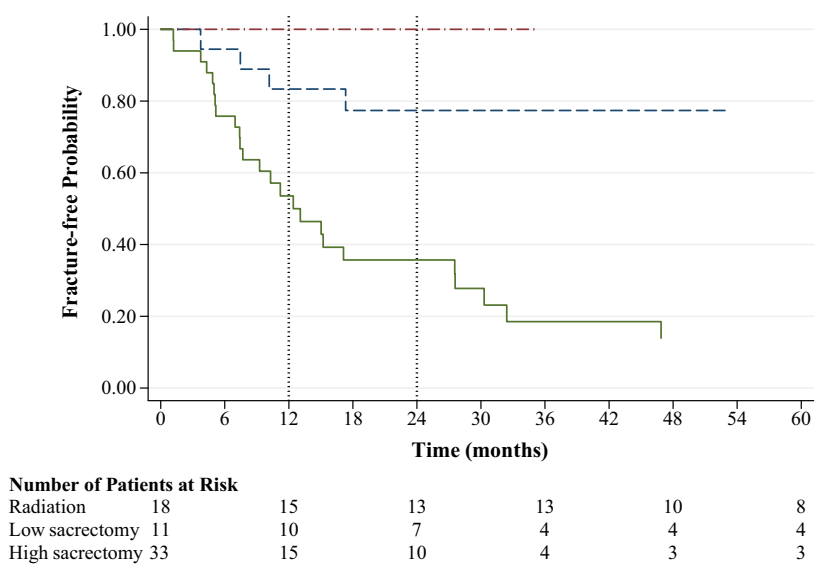

Fig. 5 Fracture-free survival probabilities in patients who had a high sacrectomy (green solid line) compared with patients who had a low sacrectomy (red dot-dashed line), and patients who had radiation patients (blue dashed line) are shown. 


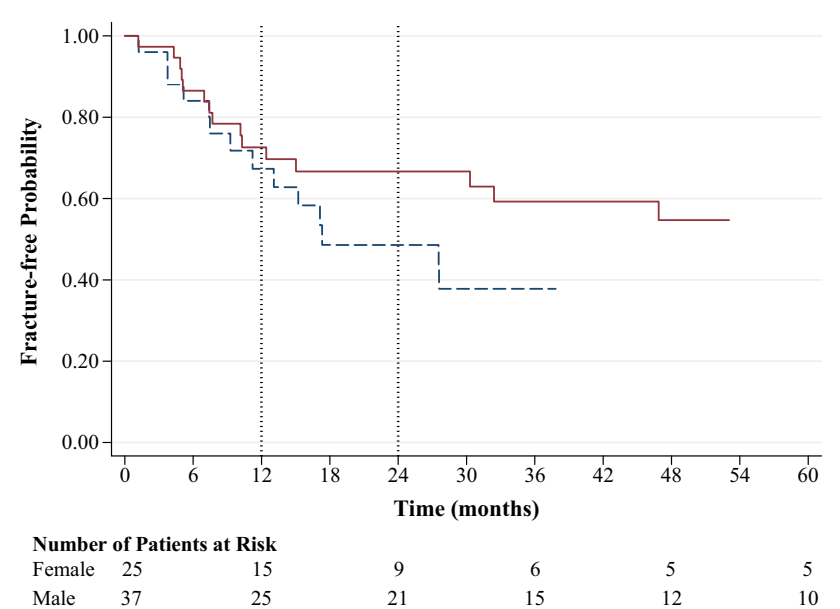

Fig. 6 Fracture-free survival probabilities for males (red solid line) compared with females (blue dashed line) are shown.

as reported in previous studies, but also can be asymptomatic and we therefore see this as an important limitation $[3,7,8,12,18]$. Third, six of the 62 patients (all surgical patients) were lost to followup by 2 years, therefore it is possible that the fracture rate in the surgery group is greater than identified in our study. Furthermore, the 2-year lost to followup rate was $27 \%$ (three patients) in the low sacrectomy group and 9\% (three patients) in the high sacrectomy group. We might have missed insufficiency fractures among patients lost to followup, which would have especially affected the fracture rate in the low sacrectomy group. Therefore, our comparison of insufficiency fracture rates among treatment groups should be considered in light of the loss to followup, and we see this as an important limitation. Fourth, the presence of a sacral insufficiency fracture was assessed by a senior radiologist (MB) based on MRI or CT and was subject to interobserver variation. However, we believe this to be independent of treatment group and therefore see this as a minor limitation. Fifth, we did not assess subsequent treatment of these fractures and corresponding outcomes. Sixth, we did not have bone mineral density measurements for our patients, and cannot account for any effect attributable to variations in bone density. Age-related osteopenia is less common among male patients and likely plays less of a role in our predominantly male patient population. To that effect, we did not find any differences in the 5-year fracture-free survival between males and females [22].

When focusing on our patients who received high-dose radiation alone, we found that approximately $1 / 4(22 \%)$ had a sacral insufficiency fracture. This is high compared with the previously reported incidence (3.1 to $11.4 \%$ ) of pelvic insufficiency fractures after radiation [9-11, 15, 17]. However, radiation regimens in these studies differed (eg, no high-dose radiation) from ours and those studies focused on radiation to visceral tumors in the pelvis rather than primary bone tumors. Radiation is a recognized risk factor for the development of insufficiency fractures; it decreases bone strength and the number and resiliency of trabeculae $[5,19,21]$. It seems plausible that higher doses of radiation would increase the risk of a fracture developing. However, the discrepancy also could be explained by the difference in definition of an insufficiency fracture among studies or the bone destruction by the chordoma in our study.

We found a higher rate of insufficiency fractures after surgery with high-dose radiation compared with radiation alone, and high sacrectomy accounted for all fractures in the surgery group. Two previous studies $[6,14]$ noted a $6 \%$ incidence of insufficiency fractures after resection of sacral chordomas (no high-dose radiation). However, insufficiency fractures were not specifically studied and the reported fracture rates therefore might be an underestimation [6, 14]. Ruggieri et al. [14] reported three insufficiency fractures (all after high sacrectomies) in 56 patients who underwent resection only, 37 of which were high sacrectomies. This translates to a fracture rate of $8.1 \%$ (three of 37) after high sacrectomy and none (0 of 19) after low sacrectomy in their cohort with a minimum followup of 3 years. This is in line with our finding that insufficiency fractures are more common after high resections compared with low resections. Fuchs et al. [6] reported three insufficiency fractures in 52 patients; 30 who underwent resection only and 22 who had resection plus radiation. Two of the fractures occurred after resection with radiation (9\%) and one occurred after resection only (3\%). We are not aware of any study that is comparable to ours and addresses insufficiency fractures after high-dose radiation for sacral tumors, as surgical resection with wide margins and no radiation is the standard of care in most institutions $[16,23]$. Conventional radiation is added in subtotal excisions or if the resection was marginal $[16,23]$.

Acknowledging the limitations of potential differences in baseline and followup among treatment groups in our study, we found that nearly $1 / 2$ of our patients had an insufficiency fracture after high-dose radiation for a sacral chordoma. The fracture rate was greater in the surgery with radiation group compared with the radiation alone group and high sacrectomy accounted for all fractures in the surgery group. These findings can be used to inform patients and also support the need for further research to elucidate the influence of high-dose radiation on bone quality.

Acknowledgments We thank the family of Stephen L. Harris for funding the "Stephen L. Harris Center for Chordoma Care" at Massachusetts General Hospital. 


\section{References}

1. Allison PD. Survival Analysis Using SAS: A Practical Guide. $2^{\text {nd }}$ ed. Cary, NC: SAS Institute Inc; 2010.

2. Chen YL, Liebsch N, Kobayashi W, Goldberg S, Kirsch D, Calkins G, Childs S, Schwab J, Hornicek F, DeLaney T. Definitive highdose photon/proton radiotherapy for unresected mobile spine and sacral chordomas. Spine (Phila Pa 1976). 2013;38:E930-936.

3. Dasgupta B, Shah N, Brown H, Gordon TE, Tanqueray AB, Mellor JA. Sacral insufficiency fractures: an unsuspected cause of low back pain. Br J Rheumatol. 1998;37:789-793.

4. DeLaney TF, Liebsch NJ, Pedlow FX, Adams J, Dean S, Yeap BY, McManus P, Rosenberg AE, Nielsen GP, Harmon DC, Spiro IJ, Raskin KA, Suit HD, Yoon SS, Hornicek FJ. Phase II study of high-dose photon/proton radiotherapy in the management of spine sarcomas. Int J Radiat Oncol Biol Phys. 2009;74:732-739.

5. Dhakal S, Chen J, McCance S, Rosier R, O'Keefe R, Constine LS. Bone density changes after radiation for extremity sarcomas: exploring the etiology of pathologic fractures. Int J Radiat Oncol Biol Phys. 2011;80:1158-1163.

6. Fuchs B, Dickey ID, Yaszemski MJ, Inwards CY, Sim FH. Operative management of sacral chordoma. J Bone Joint Surg Am. 2005;87:2211-2216.

7. Galbraith JG, Butler JS, Blake SP, Kelleher G. Sacral insufficiency fractures: an easily overlooked cause of back pain in the ED. Am J Emerg Med. 2011;29:359 e5-6.

8. Grasland A, Pouchot J, Mathieu A, Paycha F, Vinceneux P. Sacral insufficiency fractures: an easily overlooked cause of back pain in elderly women. Arch Intern Med. 1996;156:668-674.

9. Herman MP, Kopetz S, Bhosale PR, Eng C, Skibber JM, Rodriguez-Bigas MA, Feig BW, Chang GJ, Delclos ME, Krishnan S, Crane CH, Das P. Sacral insufficiency fractures after preoperative chemoradiation for rectal cancer: incidence, risk factors, and clinical course. Int J Radiat Oncol Biol Phys. 2009;74:818-823.

10. Ikushima H, Osaki K, Furutani S, Yamashita K, Kishida Y, Kudoh T, Nishitani H. Pelvic bone complications following radiation therapy of gynecologic malignancies: clinical evaluation of radiation-induced pelvic insufficiency fractures. Gynecol Oncol. 2006;103:1100-1104.

11. Kim HJ, Boland PJ, Meredith DS, Lis E, Zhang Z, Shi W, Yamada YJ, Goodman KA. Fractures of the sacrum after chemoradiation for rectal carcinoma: incidence, risk factors, and radiographic evaluation. Int J Radiat Oncol Biol Phys. 2012;84: 694-699.

12. Lin J, Lachmann E, Nagler W. Sacral insufficiency fractures: a report of two cases and a review of the literature. $J$ Womens Health Gend Based Med. 2001;10:699-705.

13. Osaka S, Toriyama S. Treatment and prognosis of sacrococcygeal chordoma: study of bone tumor registry in Japan. Nihon Seikeigeka Gakkai Zasshi. 1989;63:240-244.

14. Ruggieri P, Angelini A, Ussia G, Montalti M, Mercuri M. Surgical margins and local control in resection of sacral chordomas. Clin Orthop Relat Res. 2010;468:2939-2947.

15. Schmeler KM, Jhingran A, Iyer RB, Sun CC, Eifel PJ, Soliman PT, Ramirez PT, Frumovitz M, Bodurka DC, Sood AK. Pelvic fractures after radiotherapy for cervical cancer: implications for survivors. Cancer. 2010;116:625-630.

16. Schwab JH, Healey JH, Rose P, Casas-Ganem J, Boland PJ. The surgical management of sacral chordomas. Spine (Phila $\mathrm{Pa}$ 1976). 2009;34:2700-2704.

17. Shih KK, Folkert MR, Kollmeier MA, Abu-Rustum NR, Sonoda Y, Leitao MM Jr, Barakat RR, Alektiar KM. Pelvic insufficiency fractures in patients with cervical and endometrial cancer treated with postoperative pelvic radiation. Gynecol Oncol. 2013;128: 540-543.

18. Stroebel RJ, Ginsburg WW, McLeod RA. Sacral insufficiency fractures: an often unsuspected cause of low back pain. $J$ Rheumatol. 1991;18:117-119.

19. Sugimoto M, Takahashi S, Toguchida J, Kotoura Y, Shibamoto Y, Yamamuro T. Changes in bone after high-dose irradiation: biomechanics and histomorphology. J Bone Joint Surg Br. 1991;73: 492-497.

20. Ugurluer G, Akbas T, Arpaci T, Ozcan N, Serin M. Bone complications after pelvic radiation therapy: evaluation with MRI. $J$ Med Imaging Radiat Oncol. 2014;58:334-340.

21. Wernle JD, Damron TA, Allen MJ, Mann KA. Local irradiation alters bone morphology and increases bone fragility in a mouse model. J Biomech. 2010;43:2738-2746.

22. Wright VJ. Osteoporosis in men. J Am Acad Orthop Surg. 2006;14:347-353.

23. York JE, Kaczaraj A, Abi-Said D, Fuller GN, Skibber JM, Janjan NA, Gokaslan ZL. Sacral chordoma: 40-year experience at a major cancer center. Neurosurgery. 1999;44:74-79; discussion 79-80. 